\title{
Oligomorphic permutation groups
}

\author{
Peter J. Cameron \\ School of Mathematical Sciences \\ Queen Mary, University of London \\ London E1 4NS \\ U.K.
}

\begin{abstract}
A permutation group $G$ (acting on a set $\Omega$, usually infinite) is said to be oligomorphic if $G$ has only finitely many orbits on $\Omega^{n}$ (the set of $n$-tuples of elements of $\Omega$ ). Such groups have traditionally been linked with model theory and combinatorial enumeration; more recently their group-theoretic properties have been studied, and links with graded algebras, Ramsey theory, topological dynamics, and other areas have emerged.

This paper is a short summary of the subject, concentrating on the enumerative and algebraic aspects but with an account of grouptheoretic properties. The first section gives an introduction to permutation groups and to some of the more specific topics we require, and the second describes the links to model theory and enumeration. We give a spread of examples, describe results on the growth rate of the counting functions, discuss a graded algebra associated with an oligomorphic group, and finally discuss group-theoretic properties such as simplicity, the small index property, and "almost-freeness".
\end{abstract}

\section{Introduction}

Despite the history and importance of group theory, we have very little idea what an arbitrary group looks like. We have made important strides in understanding finite groups, by determining the finite simple groups; but we can only study general groups under some very strong condition, usually a 
finiteness condition. We have theories of finitely generated groups, locally finite groups, residually finite groups, groups of finite cohomological dimension, linear groups, profinite groups, and so forth, but no theory of general groups.

Oligomorphic groups satisfy a rather different kind of finiteness condition; paradoxically, one which makes them "large" rather than "small". A permutation group $G$ (a subgroup of the symmetric group on a set $\Omega$ is said to be oligomorphic if $G$ has only finitely many orbits on $\Omega^{n}$ for every natural number $n$. (An element of $G$ acts componentwise on the set $\Omega^{n}$ of all $n$-tuples of points of $\Omega$.)

Thus, by definition, an oligomorphic group $G$ gives us a sequence of natural numbers, the numbers of orbits on $n$-tuples for $n=0,1,2, \ldots$ Not surprisingly, the theory is connected with counting problems in various parts of mathematics (combinatorics, model theory, graded algebras). Curiously, there is rather less we can say about the groups themselves. If $G$ is oligmorphic and $H$ is a proper subgroup having the same orbits on $n$-tuples as $G$ for all $n$, then counting cannot distinguish between $G$ and $H$, even though they may be very different as groups (for example, $G$ may be simple while $H$ is a free group).

In the remainder of this section, we introduce some basics of permutation group theory and of the counting functions associated wiith oligomorphic groups. For further information about permutation groups, see [7, 14]. Note also that there are many connections between parts of the theory of oligomorphic groups and that of (combinatorial) species, as developed by Joyal [21].

\subsection{Permutation groups}

This section is a brief introduction to permutation groups. For more details, see [7].

The symmetric group $\operatorname{Sym}(\Omega)$ on a set $\Omega$ is the group of all permutations of $\Omega$. If $\Omega$ is finite, say $\Omega=\{1,2, \ldots, n\}$, we write the symmetric group as $S_{n}$. We write permutations on the right, so that the image of $\alpha$ under $g$ is written $\alpha g$.

An orbit of $G$ is an equivalence class of the relation $\alpha \sim \beta$ if $\alpha g=\beta$ for some $g \in G$; in other words, a set of the form $\{\alpha g: g \in G\}$. We say that $G$ is transitive if it has only one orbit. In a sense, any permutation group can be "resolved" into transitve groups.

There is a partial converse. If $G_{i}$ is a transitive permutation group on $\Omega_{i}$ 
for $i \in I$, where $I$ is some index set, the cartesian product $\prod_{i \in I} G_{i}$ is the set of functions $f: I \rightarrow \bigcup_{i \in I} G_{i}$ satisfying $f(i) \in G_{i}$ for all $i \in I$. It has two natural actions:

- The intransitive action on the disjoint union of the sets $\Omega_{i}$ : if $\alpha \in \Omega_{i}$, then $\alpha f=\alpha f(i)$. If each group $G_{i}$ is transitive, then the sets $\Omega_{i}$ are the orbits of the Cartesian product.

- There is also a product action, componentwise on the Cartesian product of the sets $\Omega_{i}$.

If $I$ is finite, we speak of the direct product, and write it as (for example) $G_{1} \times \cdots \times G_{k}$, if $I=\{1, \ldots, k\}$.

Thus, if $G_{1}=S_{n}$ and $G_{2}=S_{m}$, then $G_{1} \times G_{2}$ has an intransitive action on $m+n$ points, and a product action on $m n$ points (which can be regarded as a rectangular grid with $G_{1}$ permuting the rows and $G_{2}$ the columns).

Now let $G$ be transitive on $\Omega$. A congruence is an equivalence relation on $\Omega$ which is $G$-invariant. There are two "trivial" congruences on $\Omega$ : the relation of equality, and the "universal" relation with a single equivalence class. We say that $G$ is primitive if there are no other congruences. For example, the symmetric group is primitive.

Important exampes of imprimitive groups are the wreath products, defined as follows. Let $H$ be a permutation group on $\Gamma$, and $K$ a permutation group on $\Delta$. Let $\Omega=\Gamma \times \Delta$, regarded as a union of copies of $\Gamma$ indexed by $\Delta$ : thus $\Gamma_{\delta}=\{(\gamma, \delta): \gamma \in \Gamma\}$, for each $\delta \in \Delta$. Take a copy $H_{\delta}$ of $H$ for each $\delta \in \Delta$, where $H_{\delta}$ acts on $\Gamma_{\delta}$. Then the Cartesian product $B=\prod_{\delta \in \Delta} H_{\delta}$ acts on $\Omega$ (in its intransitive action). Moreover, the group $K$ acts on $\Omega$ by permuting the second components (i.e. by permuting the "fibres" $\Gamma_{\delta}$. The wreath product $G=H \mathrm{Wr} K$ is the group generated by $B$ and $K$; we call $B$ the base group (it is a normal subgroup) and $K$ the top group of the wreath product.

We note that there are different notations and conventions in other areas of mathematics. For example, in experimental design in statistics [2], direct product (product action) is called crossing, and wreath product is called nesting, but nesting is written with the top structure before the bottom one, e.g. $\Delta / \Gamma$ in our case.

If $|\Gamma|>1$ and $|\Delta|>1$, the wreath product is imprimitive: the relation $(\gamma, \delta) \equiv\left(\gamma^{\prime}, \delta^{\prime}\right)$ if $\delta=\delta^{\prime}$ is a congruence. For this reason it is called the 
imprimitive action. Any imprimitive permutation group can be embedded in a wreath product in a natural way.

There is another action of the wreath product, the power action, on the set $\Gamma^{\Delta}$ of functions from $\Delta$ to $\Gamma$. It bears a similar relation to the imprimitive action as the product action does to the intransitive action for the Cartesian product. If we regard the domain of the imprimitive action as a fibred space, with fibres $\Gamma_{\delta}$ isomorphic to $\Gamma$ indexed by $\Delta$, then the domain for the power action is the set of global sections (subsets containing one point from each fibre).

\subsection{Oligomorphic permutation groups}

For a natural number $n$, a permutation group is $n$-transitive if it acts transitively on the set of $n$-tuples of distinct elements of $\Omega$, and $n$-set-transitive if it acts transitively on the set of $n$-element subsets of $\Omega$. We say a permutation group is highly transitive if it is $n$-transitive for all $n$, and highly set-transitive if it is $n$-set-transitive for all $n$.

Oligomorphic permutation groups generalise these classes. Thus, we let $F_{n}(G)$ denote the number of orbits of $G$ on the set of $n$-tuples of distinct elements, and $f_{n}(G)$ the number of orbits on $n$-element subsets. So $G$ is $n$-transitive (resp. $n$-set-transitive) if $F_{n}(G)=1$ (resp. $\left.f_{n}(G)=1\right)$. If the group $G$ is clear, we drop it and write simply $F_{n}, f_{n}$.

The definition speaks of orbits on $\Omega^{n}$, the set of all $n$-tuples. Let $F_{n}^{*}(G)$ denote the number of these orbits. It is clear that, for given $n$, one of these numbers is finite if and only if the others are; indeed, we have

- $F_{n}^{*}=\sum_{k=1}^{n} S(n, k) F_{k}$, where $S(n, k)$ is the Stirling number of the second kind (the number of partitions on an $n$-set into $k$ parts);

- $f_{n} \leq F_{n} \leq n ! f_{n}$.

As an example for the first point, if $G$ is highly transitive, then

$$
F_{n}^{*}(G)=\sum_{k=0}^{n} S(n, k)=B(n)
$$

the $n$th Bell number (the number of partitions of an $n$-set).

In the second point, both bounds are attained: 
- Let $G=\operatorname{Sym}(\Omega)$ (we will always denote this group by $S$ ). Then $f_{n}(S)=F_{n}(S)=1$ for all $n$.

- Let $G$ be the group of order-preserving permutations of the rational numbers (we will always denote this group by $A$ ). We can map any $n$ rational numbers in increasing order to any other $n$ such by an orderpreserving permutation (by filling in the gaps to produce a piecewiselinear map); so $f_{n}(A)=1$ and $F_{n}(A)=n$ !.

Highly set-transitive groups must resemble the above types. All we can do is to modify the total order slightly. The following is proved in [3].

Theorem 1.1 Let $G$ be a highly set-transitive but not highly transitive permutation group. Then there is a linear or circular order preserved by $G$. In particular, $G$ is not 4-transitive.

\subsection{Topology}

We will only consider permutation groups of countable degree in this article. If we are interested in the sequences $f_{n}$ and $F_{n}$, this is justified by the following result, which follows from the Downward Löwenheim-Skolem Theorem of model theory:

Proposition 1.2 Let $G$ be an oligomorphic permutation group on an infinite set. Then there is an oligomorphic permutation group $G^{\prime}$ on a countably infinite set such that $F_{n}\left(G^{\prime}\right)=F_{n}(G)$ and $f_{n}\left(G^{\prime}\right)=f_{n}(G)$ for all $n \in \mathbb{N}$.

There is a natural topology on the symmetric group of countable degree. This is the topology of pointwise convergence, where a sequence $\left(g_{n}\right)$ converges to $g$ if $\alpha_{i} g_{n}=\alpha_{i} g$ for all sufficiently large $n$, where $\left(\alpha_{1}, \alpha_{2}, \ldots\right)$ is an enumeration of $\Omega$. This topology can be derived from a complete metric. The topology is specified by the first part of the proposition below.

Proposition $1.3 \quad$ (a) $G$ is open in $\operatorname{Sym}(\Omega)$ if and only if it contains the pointwise stabiliser of a finite set.

(b) $G$ is closed in $\Omega$ if and only if $G$ is the automorphism group of a relational structure on $\Omega$, that is, a family of relations (of various arities) on $\Omega$. 
The closure of a permutation group $G$ consists of all permutations which preserve the $G$-orbits on $\Omega^{n}$ for all $n$. We remarked earlier that, as far as orbit-counting goes, we cannot distinguish between groups with the same orbits. The larges such group is necessarily closed in $\operatorname{Sym}(\Omega)$. So we may restrict our attention to closed groups of countable degree (that is, automorphism groups of countable relational structures) if we are interested in orbit-counting.

The fact that the topology on the symmetric group is derived from a complete metric means that the same is true for any closed subgroup. This permits use of the Baire category theorem. Recall that a subset of a complete metric space is residual if it contains the intersection of countably many open dense subsets. The Baire category theorem asserts that a residual set is non-empty. Indeed, residual sets are "large" (for example, they have non-empty intersection with any open set, and the intersection of countably many residual sets is residual). Often it is possible to give a non-constructive existence proof for some object by showing that objects of the required type form a residual set.

\subsection{Cycle index}

An important tool in combinatorial enumeration is the cycle index of a finite permutation group $G$, which is the polynomial in the indeterminates $s_{1}, s_{2}, \ldots, s_{n}$ (where $n$ is the degree) given by

$$
Z\left(G ; s_{1}, s_{2}, \ldots, s_{n}\right)=\frac{1}{|G|} \sum_{g \in G} s_{1}^{c_{1}(g)} s_{2}^{c_{2}(g)} \cdots s_{n}^{c_{n}(g)},
$$

where $c_{i}(g)$ is the number of $i$-cycles in the decomposition of the permutation $g$ into disjoint cycles.

For its role in enumeration, see [18].

One cannot simply take this definition unchanged for infinite permutation groups, for several reasons: the number $c_{i}(g)$ may be infinite; there may be infinite cycles; and the denominator $|G|$ is infinite.

The trick to generalising it lies in the following result about finite groups, the Shift Lemma. Let $\mathcal{P} \Omega$ be the set of all subsets of the finite set $\Omega$, and let $\mathcal{P} \Omega / G$ denote a set of representatives on the $G$-orbits on $\mathcal{P} \Omega$. Also, if $X$ is any subset of $\Omega$, we let $G[X]$ denote the permutation group on $X$ induced by its setwise stabiliser in $G$. 
Proposition 1.4 For a finite permutation group $G$ on $\Omega$,

$$
\sum_{X \in \mathcal{P} \Omega / G} Z\left(G[X] ; s_{1}, s_{2}, \ldots\right)=Z\left(G ; s_{1}+1, s_{2}+1, \ldots\right) .
$$

Now let $G$ be any oligomorphic permutation group on the (possibly infinite) set $\Omega$. We define the modified cycle index of $G$ to be the left-hand side of the Shift Lemma, with one small modification: we replace $\mathcal{P} \Omega$ by $\mathcal{P}_{\text {fin }} \Omega$, the set of all finite subsets of $\Omega$. Thus, the modified cycle index is

$$
\tilde{Z}\left(G ; s_{1}, s_{2}, \ldots\right)=\sum_{X \in \mathcal{P}_{\mathrm{fin}} \Omega / G} Z\left(G[X] ; s_{1}, s_{2}, \ldots\right) .
$$

Each term in the sum is the cycle index of a finite permutation group. Also since $G$ is oligomorphic, there are only a finite number of terms in the sum which contribute to the coefficient of a fixed monomial $s_{1}^{c_{1}} \cdots s_{r}^{c_{r}}$, namely those corresponding to the $f_{n}(G)$ orbits on sets of cardinality $n=c_{1}+$ $2 c_{2}+\cdots+r c_{r}$. (We see here that the definition would fail if $G$ were not oligomorphic.) So we have defined a formal power series in $s_{1}, s_{2}, \ldots$ We also see that if $G$ happens to be a finite permutation group, then we have the ordinary cycle index with 1 added to each indeterminate.

Our previous counting functions can be extracted from the modified cycle index:

Proposition 1.5 Let $G$ be an oligomorphic permutation group.

- $F_{G}(x)$ is obtained from $\tilde{Z}(G)$ by substituting $s_{1}=x$ and $s_{i}=0$ for $i>1$.

- $f_{G}(x)$ is obtained from $\tilde{Z}(G)$ by substituting $s_{i}=x^{i}$ for all $i$.

Just as before, it is true that

- for any oligomorphic permutation group $G$, there is an oligomorphic group $G^{\prime}$ of countable degree satisfying $\tilde{Z}(G)=\tilde{Z}\left(G^{\prime}\right)$;

- an oligomorphic group of countable degree and its closure have the same modified cycle index.

So we may consider closed groups of countable degree.

We conclude this section by displaying the modified cycle index for the groups $S$ (the infinite symmetric group) and $A$ (the group of order-preserving permutations of $\mathbb{Q}$ ). 


\section{Proposition 1.6}

$$
\begin{aligned}
& \tilde{Z}(S)=\exp \left(\sum_{i \geq 1} \frac{s_{i}}{i}\right) ; \\
& \tilde{Z}(A)=\frac{1}{1-s_{1}} .
\end{aligned}
$$

\section{Connections}

Oligomorphic permutation groups are closely connected with two other areas of mathematics: model theory, and combinatorial enumeration. In this section we discuss the connections.

\subsection{Model theory}

Model theory describes structures consisting of a set with a collection of constants, relations and functions. Much of mathematics can be fitted into this framework, often in different ways. For example, a group has a binary operation (composition), a unary operation (inversion), and a constant (the identity); but the second and third may be defined in terms of the first. A graph can be regarded as a set of vertices with a binary relation (adjacency), or as a set of vertices and edges with a unary relation (to distinguish the vertices) and a binary operation of incidence. The latter is appropriate for multigraphs.

Logic describes such structures by means of collections of formulae. The language includes symbols for the relations, functions, and constants, connectives and quantifiers, equality, brackets, and a supply of variables. We work in first-order logic: we are allowed to combine finitely many formulae with connectives, and to quantify over variables which range over the underlying set. I will assume that the language is countable. A sentence is a formula with no free variables (all variables are quantified). Thus for example the group axioms can be expressed as a single sentence (the conjunction of the associative, identity and inverse laws, each universally quantified over all variables).

A theory is a set of sentences; a structure is a model for a theory if every sentence in the theory is true in the model. 
Sometimes we want a theory to have a wide range of models (this is the case with group theory). At other times, we have a particular model in mind, and want to capture as much of its essence as possible in a theory (this is the case with the Peano axioms for the natural numbers). It is known that, as long as a theory has infinite models, it cannot have a unique model; there will be models of arbitrarily large cardinality. The best we can do is ask that the theory is $\alpha$-categorical, where $\alpha$ is an infinite cardinal, meaning that there is a unique model of cardinality $\alpha$ up to isomorphism. By a theorem of Vaught, there are only two types of categoricity, countable and uncountable: if a theory is $\alpha$-categorical for some uncountable cardinal $\alpha$, then it is $\alpha$ categorical for all such.

Uncountable categoricity gives rise to a powerful structure theory, extending that for vector spaces over a fixed field or algebraically closed fields of fixed characteristic (where a single invariant, the rank, determines the model). By contrast, countable categoricity is related to symmetry, by the following remarkable theorem due independently to Engeler, Ryll-Nardzewski and Svenonius:

Theorem 2.1 Let $M$ be a countable model of a theory $T$ over a countable language. Then $T$ is $\aleph_{0}$-categorical if and only if the automorphism group of $M$ is oligomorphic.

In fact, if $M$ is the unique countable model of $T$, and $G=\operatorname{Aut}(M)$, then $F_{n}^{*}(T)$ is the number of $n$-types over $T$ (maximal consistent sets of formulae in $n$ free variables). So our results are applicable to counting types in such theories.

Note in passing that the automorphism group of $\mathbb{N}$ is trivial; so the Peano axioms have countable "non-standard" models.

\subsection{Combinatorial enumeration}

The set-up is similar, but we restrict ourselves to relational structures (no function or constant symbols). We will see that the counting problems for orbits on sets and tuples of oligomorphic permutation groups are identical with those for unlabelled and labelled structures in so-called oligomorphic Fraïssé classes of relational structures. These include large numbers of combinatorially important classes of structures, so we have a rather general paradigm for interesting counting problems. 
Take a fixed relational language $\mathbf{L}$ (this means, each set carries named relations of prescribed arities). For example, we could consider graphs, directed graphs, tournaments, partially ordered sets, $k$-edge-coloured graphs, graphs with a fixed bipartition, or much more recondite examples.

A Fraïssé class over $\mathbf{L}$ is a class $\mathcal{C}$ of finite relational structures over $\mathbf{L}$ satisfying the following four conditions:

(a) $\mathcal{C}$ is closed under isomorphism;

(b) $\mathcal{C}$ is closed under taking induced substructures (this means, take a subset of the domain, and all instances of all relations which are contained within this subset);

(c) $\mathcal{C}$ has only countably many members up to isomorphism;

(d) $\mathcal{C}$ has the amalgamation property: this means that, if $B_{1}, B_{2} \in \mathcal{C}$ have isomorphic substructures, they can be "glued together" along these substructures (or possibly more) to form a structure in $\mathcal{C}$. Formally, if $A, B_{1}, B_{2} \in \mathcal{C}$ and $f_{i}: A \rightarrow B_{i}$ is an embedding for $i=1,2$, then there is a structure $C \in \mathcal{C}$ and embeddings $h_{i}: B_{i} \rightarrow C$ for $i=1,2$ so that $h_{1} f_{1}=h_{2} f_{2}$.

(The reader is warned that I allow the structure $A$ to be empty here. Some authors exclude this, state the special case where $A=\emptyset$ separately, and call it the joint embedding property.)

For a simple example, finite graphs form a Fraïssé class: take the union of $B_{1}$ and $B_{2}$ identifying the isomorphic substructures, and put any or no edges between $B_{1} \backslash A$ and $B_{2} \backslash A$. It is just a little more difficult to show that finite total or partial orders form a Fraïssé class.

Let $M$ be a structure over $\mathbf{L}$. We make two definitions:

- The age of $M$ is the class of all finite $\mathbf{L}$-structures which are embeddable in $M$.

- $M$ is homogeneous if any isomorphism between finite induced substructures of $M$ can be extended to an automorphism of $M$.

For example, the totally ordered set $\mathbb{Q}$ is homogeneous, and its age consists of all finite totally ordered sets.

Fraïssé's Theorem asserts: 
Theorem 2.2 A class $\mathcal{C}$ of finite $\mathbf{L}$-structures is the age of a countable homogeneous $\mathbf{L}$-structure $M$ if and only if it is a Fraïssé class. If these conditions hold, then $M$ is unique up to isomorphism.

We call the countable homogeneous structure $M$ the Fraïssé limit of the class $\mathcal{C}$. Thus, $(\mathbb{Q},<)$ is the Fraïssé limit of the class of finite totally ordered sets. The Fraïssé limit of the class of finite graphs is the celebrated random graph, or Rado graph $R$, which is extensively discussed in [4].

Now suppose that a Fraïssé class satisfies the following stronger version of condition (c):

$\left(\mathrm{c}^{\prime}\right)$ For any $n \in \mathbb{N}$, the class $\mathcal{C}$ has only finitely many $n$-element members up to isomorphism.

We call such a class an oligomorphic Fraïssé class. All examples mentioned so far are oligomorphic; the condition certainly holds if $\mathbf{L}$ contains only finitely many relations. Let $M$ be its Fraïssé limit, and let $G=\operatorname{Aut}(M)$ be the automorphism group of $M$. By homogeneity, it follows that

- $F_{n}(G)$ is the number of labelled $n$-element structures in $\mathcal{C}$ (that is, structures on the set $\{1,2, \ldots, n\}$;

- $f_{n}(G)$ is the number of unlabelled $n$-element structures in $\mathcal{C}$ (that is, isomorphism classes of $n$-element structures).

Some Fraïssé classes satisfy a stronger version of the amalgamation property, called strong amalgamation. This is said to hold if the amalgam of any two structures can be produced without identifying any points not in the common substructure. More formally, if $h_{1}\left(b_{1}\right)=h_{2}\left(b_{2}\right)$, then $b_{1}=f_{1}(a)$ and $b_{2}=f_{2}(a)$ for some $a \in A$.

This holds in the above examples. An example where it fails is given by the class of graphs consisting of isolated vertices and edges. If $B_{1}$ and $B_{2}$ are edges and $A$ a single vertex, then in the amalgam we are forced to identify the other ends of the two edges as well.

Proposition 2.3 A Fraïssé class has the strong amalgamation property if and only if the automorphism group of its Fraïsé limit has the property that the stabiliser of any finite set of points fixes no additional points.

Later we will see a still stronger version. 


\section{Constructions}

There are two main sources for examples of (closed) oligomorphic groups: building new examples from old, or constructing Fraïssé classes (the group is then the automorphism group of the Fraïssé limit of the class).

\subsection{Direct and wreath products}

Suppose that $G_{1}$ and $G_{2}$ are oligomorphic permutation groups on $\Omega_{1}$ and $\Omega_{2}$ respectively. We can form their direct or wreath product, and each of these has two actions, which we now discuss.

Direct product, intransitive action The direct product $G=G_{1} \times G_{2}$ acts on the disjoint union of $\Omega_{1}$ and $\Omega_{2}$, say $\Omega$. An $n$-subset of $\Omega$ consists of $k$ points of $\Omega_{1}$ and $n-k$ points of $\Omega_{2}$, for some $k$ with $0 \leq k \leq n$; two $n$-sets are in the same $G$-orbit if and only if their intersections with $\Omega_{i}$ are in the same $G_{i}$-orbit for $i=1,2$. Thus $\left(f_{n}(G)\right)$ is the convolution of $\left(f_{n}\left(G_{1}\right)\right)$ and $\left(f_{n}\left(G_{2}\right)\right)$ :

$$
f_{n}(G)=\sum_{k=0}^{n} f_{k}\left(G_{1}\right) f_{n-k}\left(G_{2}\right),
$$

from which it follows that the generating functions multiply:

$$
f_{G}(x)=f_{G_{1}}(x) f_{G_{2}}(x) .
$$

Similarly, for $F_{n}$, we have an exponential convolution, so that the exponential generating functions also multiply:

$$
F_{G}(x)=F_{G_{1}}(x) F_{G_{2}}(x) .
$$

The modified cycle index is also multiplicative:

$$
\tilde{Z}(G)=\tilde{Z}\left(G_{1}\right) \tilde{Z}\left(G_{2}\right) .
$$

Direct product, product action The direct product $G_{1} \times G_{2}$ also has a product action on the Cartesian product $\Omega_{1} \times \Omega_{2}$. This is more difficult to analyse; the recent paper [9] describes the situation.

First, there is a multiplicative property: it is easy to see that

$$
F_{n}^{*}(G)=F_{n}^{*}\left(G_{1}\right) F_{n}^{*}\left(G_{2}\right) .
$$


The modified cycle index of the product can be computed as follows. Define an operation on the indeterminates by

$$
s_{i} \bullet s_{j}=\left(s_{\operatorname{lcm}(i, j)}\right)^{\operatorname{gcd}(i, j)} .
$$

Extend this operation multiplicatively to monomials and then additively to sums of monomials. Then we have

$$
\tilde{Z}(G)=\tilde{Z}\left(G_{1}\right) \bullet \tilde{Z}\left(G_{2}\right) .
$$

It should clearly be possible to deduce the first of these relations from the second; but this is surprisingly difficult (see the cited paper).

Here is an example. Consider first the group $G=A$, the order-preserving permutations of $\mathbb{Q}$. We have $F_{n}(G)=n$ !, and hence

$$
F_{n}^{*}(G)=\sum_{k=1}^{n} S(n, k) k !=P(n),
$$

the number of preorders of an $n$-set (reflexive and transitive relations).

Now let $G=A \times A$ with the product action. We have $F_{n}^{*}(G)=P(n)^{2}$, and so

$$
F_{n}(G)=\sum_{k=1}^{n} s(n, k) P(k)^{2} .
$$

Moreover, since $G$ (like $A$ ) has the property that the setwise stabiliser of an $n$-set fixes it pointwise, we have

$$
f_{n}(G)=F_{n}(G) / n !
$$

Now the exponential generating function for $P(n)$ is $1 /\left(2-\mathrm{e}^{x}\right)$, with the nearest singularity to the origin at $\log 2$; so $P(n)$ is roughly $n ! /(\log 2)^{n}$. So $F_{n}(G)$ is about $(n !)^{2} /(\log 2)^{2 n}$. Since the Stirling numbers alternate in sign, it is not completely trivial to find the asymptotics of $f_{n}(G)$. This was achieved in [10], using three entirely different methods; it turns out that

$$
f_{n}(G) \sim \frac{n !}{4} \mathrm{e}^{-\frac{1}{2}(\log 2)^{2}} \frac{1}{(\log 2)^{2 n+2}} .
$$

In broad-brush terms, "factorial times exponential". 
Wreath product, imprimitive action The wreath product $G_{1} \mathrm{Wr} G_{2}$ has its imprimitive action on $\Omega_{1} \times \Omega_{2}$; as we have seen, this should be thought of as a covering of $\Omega_{2}$ with fibres isomorphic to $\Omega_{1}$. The function $F_{G}(x)$ is found by substitution:

$$
F_{G}(x)=F_{G_{2}}\left(F_{G_{1}}(x)-1\right) .
$$

In particular, for any oligomorphic group $G$, we have

$$
F_{S W r G}(x)=F_{G}\left(\mathrm{e}^{x}-1\right)=F_{G}^{*}(x),
$$

so that $F_{n}(S \mathrm{Wr} G)=F_{n}^{*}(G)$ for all $n$, as is easily seen directly.

For another example, we note that $F_{G \mathrm{Wr} S}(x)=\exp \left(F_{G}(x)-1\right)$. Thus, the substitution rule for the wreath product can be regarded as the prototype of a wide generalisation of the exponential principle in combinatorics [33, 16]. If $G$ is the automorphism group of the Fraïssé limit of a Fraïssé class $\mathcal{C}$, then $G \mathrm{Wr} S$ is similarly associated with the class of disjoint unions of $\mathcal{C}$ structures; the exponential principle applies to this situation. In general, if $H$ is associated with a Fraïssé class $\mathcal{D}$, then $G \mathrm{Wr} H$ is associated with the class of disjoint unions of $\mathcal{C}$-structures with a $\mathcal{D}$-structure on the set of parts. For example, if $H=A$, then we have ordered sequences of $\mathcal{C}$-structures; if $H$ is the automorphism group of the random graph, we have a graphs whose vertices are $\mathcal{C}$-structures; and so on.

The numbers $f_{n}(G)$ of orbits on unordered sets cannot be obtained from the sequences $\left(f_{n}\left(G_{1}\right)\right)$ and $\left.f_{n}\left(G_{2}\right)\right)$ alone; we need the modified cycle index of the top group $G_{2}$. The generating function $f_{G}(x)$ is obtained from $\tilde{Z}\left(G_{2}\right)$ by substituting $f_{G_{1}}\left(x^{i}\right)-1$ for the variable $s_{i}$, for $i=1,2, \ldots$.

Wreath product, power action The wreath product also has a power action on $\Omega_{1}^{\Omega_{2}}$, the set of functions from $\Omega_{2}$ to $\omega_{1}$ (the set of transversals to the fibres in the imprimitive action). This action is not in general oligomorphic; it is so if $G_{2}$ is a finite group.

It is shown in [9] that $F_{n}^{*}(G)$ is obtained from the (ordinary) cycle index of the finite group $G_{2}$ by substiting $F_{n}^{*}\left(G_{1}\right)$ for all of the variables $s_{i}$.

\subsection{Other examples}

Further examples are most easily described as automorphism groups of relational structures. As we have seen, any closed oligomorphic group of countable degree is the automorphism group of a homogeneous relational structure, 
which is the Fraïssé limit of a Fraïssé class of finite structures. Sometimes the easiest way to specify the group is to give the Fraïssé class.

The random graph and some of its relations. Let $R$ be the countable random graph. This graph is the Fraïssé limit of the class of finite graphs. There are direct constructions for it, see [4]: for example, the vertices are the primes congruent to $1 \bmod 4$, and $p$ and $q$ are joined if and only if $p$ is a quadratic residue mod $q$. Alternatively, as Erdős and Rényi showed, if we form a countable random graph by choosing edges independently with probability $1 / 2$ from the 2 -subsets of a countable set, the resulting graph is isomorphic to $R$ with probability 1 .

The graph $R$ is homogeneous, and contains all finite (and indeed all countable) graphs as induced subgraphs. So, if $G=\operatorname{Aut}(R)$, then $F_{n}(G)$ is the number of labelled graphs on $n$ vertices (which is $2^{n(n-1) / 2}$ ), while $f_{n}(G)$ is the number of unlabelled graphs on $n$ vertices (which is asympptotically $F_{n}(G) / n$ !). Note that these sequences grow so fast that the generating functions converge only at the origin.

There are several related Fraïssé classes. Here are a few examples.

- In place of graphs, we can take directed graphs, or oriented graphs, or tournaments, or $k$-uniform hypergraphs. Note that the automorphism group of the random $k$-uniform hypergraph is $(k-1)$-transitive but not $k$-transitive; so every degree of transitivity is possible for infinite permutation groups.

- Thomas [34] showed that there are only five closed supergroups of $\operatorname{Aut}(R)$, namely

$-\operatorname{Aut}(R)$

- The group $D(R)$ of dualities (automorphisms and anti-automorphisms) of $R$, which is 2-transitive and contains $\operatorname{Aut}(R)$ as a normal subgroup of index 2 ;

- The group $S(R)$ of switching automorphisms of $R$ (see below), which is 2-transitive;

- The group $B(R)$ of switching automorphisms and anti-automorphisms of $R$, which is 3-transitive and contains $S(R)$ as a normal subgroup of order 2 ; 
- the symmetric group.

- Lachlan and Woodrow [24] determined all the countable homogeneous graphs. There are some trivial ones (disjoint unions of complete graphs of the same size, and their complements); some non-trivial ones, the Henson graphs and their complements; and the random graph. The Henson graph $H_{n}$ is the Fraïssé limit of the class of all finite graphs which contain no complete graph of order $n$, for $n \geq 3$. They are not very well understood.

The operation $\sigma_{X}$ of switching a graph with respect to a set $X$ of vertices consists in replacing edges between $X$ and its complement by non-edges, and non-edges by edges, while keeping things inside or outside $X$ the same as before. A permutation $g$ is a switching automorphism of $\Gamma$ if $\Gamma^{g}=\sigma_{X}(\Gamma)$ for some set $X$. Switching anti-automorphisms are defined similarly.

One curious fact is that $F_{n}(S(R))$ and $f_{n}(S(R))$ are equal to the numbers of labelled and unlabelled even graphs on $n$ vertices (graphs with all vertex degrees even), although the even graphs do not form a Fraïssé class.

Ordered structures An old theorem of Skolem says that, if two subsets $X$ and $Y$ of $\mathbb{Q}$ are dense and have dense complements, then there is an order-preserving permutation of $\mathbb{Q}$ carrying $X$ to $Y$. More generally, consider colourings of $\mathbb{Q}$ with $m$ colours so that each colour class is dense. It is not hard to show that there is a unique such colouring up to orderpreserving permutations. The automorpism group $A_{m}$ of such a colouring (the group of permutations preserving the order and the colours) is oligomorphic, and $f_{n}\left(A_{m}\right)=m^{n}$. For, if $\left\{c_{1}, \ldots, c_{m}\right\}$ is the set of colours, then an $n$-set $\left\{q_{1}, \ldots, q_{n}\right\}$, with $q_{1}<\cdots<q_{m}$, can be described by a word of length $n$ in the alphabet $\left\{c_{1}, \ldots, c_{m}\right\}$, whose $i$ th letter is the colour of $q_{i}$; two sets lie in the same orbit if and only if they are coded by the same word, and every word arises as the code of some subset.

We can modify this example in the same way we $\operatorname{did}$ for $\mathbb{Q}$ itself, allowing ourselves to preserve or reverse the order, or turning it into a circular order.

Treelike structures There are vast numbers of treelike structures; I cannot give even a brief overview, so I will concentrate on a couple of examples.

Consider the class of boron trees, that is, finite trees in which each vertex has degree 1 or 3 . Consider any four leaves in such a tree. They are connected 
by a graph of the following shape:

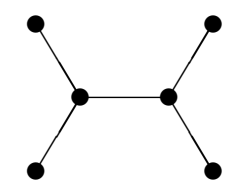

We see that one of the three partitions of the four leaves into two sets of two is distinguished by the fact that the paths joining vertices in the same pair do not intersect. Thus, there is a quaternary relation on the set of leaves. It is possible to show that this relation uniquely determines the boron tree, and that the class of finite structures carrying quaternary relations which arise in this way is a Fraïssé class. So there is a corresponding oligomorphic group $G$, which is 3-transitive but not 4-transitive, and satisfies $f_{n}(G) \sim A n^{-5 / 2} c^{n}$, where $c=2.483 \ldots$

This construction can be modified in many ways, of which a few are given below.

- We may consider trees with degrees $1,3,4, \ldots, m$, or with any possible degree except 2. (Divalent vertices are invisible in this construction.)

- We may consider internal vertices as well as leaves: there will be a ternary betweenness relation saying that one vertex is on the path joining the other two.

- By embedding the trees in the plane, we may impose a circular order on the set of leaves.

Many of these constructions give examples with exponential growth (roughly $c^{n}$ for some $c>1$ ).

Other examples The symmetric group $S$ on a countable set $\Omega$ has an induced action on the set of $k$-element subsets of $\Omega$, for any $k$. These groups are oligomorphic, but except in the case $k=2$, not much is known about the asymptotics of the orbit-counting sequences (see [11] for the case $k=2$ ).

Other examples are linear and affine groups on infinite-dimensional vector spaces over finite fields. See [12] for the asymptotics. 


\section{Growth rates}

In this section we survey some known results about the rate of growth of the sequences $\left(f_{n}(G)\right)$ and $\left(F_{n}(G)\right)$ for an oligomorphic group $G$.

First, note that there is no upper bound for growth rates. Given any sequence $\left(a_{n}\right)$ of natural numbers, let $\mathbf{L}$ be a relational language containing $a_{n} n$-ary relations $\mathcal{C}$ be the Fraïssé class consisting of all structures in which an $n$-ary relation holds only if all its arguments are distinct. If $G$ is the automorphism group of the Fraïssé limit, then $f_{n}(G) \geq 2^{a_{n}}$ for all $n$. Indeed, it is slow growth which is most interesting!

Foe intransitive or imprimitive groups, we can have polynomial growth for $\left(f_{n}\right)$. For a simple example, if $G$ is the direct product of $r$ symmetric groups (with the intransitive action), then $f_{n}(G)=\left(\begin{array}{c}n+r-1 \\ r-1\end{array}\right)$, with generating function $f_{G}(x)=(1-x)^{-r}$.

For the same group, $F_{n}(G)=r^{n}$, since the orbit of an $n$-tuple is determined by the orbit containing each of its points. Recently it has been shown [1] that, if $G$ is transitive, and the point stabiliser has $m$ orbits on the remaining points, then $F_{n}(G) \geq m^{n}$. Equality is possible here for any $m$.

There is a gap above polynomial growth for $\left(f_{n}\right)$. The next possible growth rate is fractional exponential, about $\exp (\sqrt{n})$. This is realised by the group $G=S \mathrm{Wr} S$, for which we have $f_{n}(G)=p(n)$, the number of partitions of the integer $n$ (with growth asymptotically ...), and $F_{n}(G)=B(n)$, the number of partitions of an $n$-set (the Bell number), whose growth is faster than exponential but slower than factorial.

However, if we insist that the group is primitive, there is a dramatic change $[25,29]$ :

Theorem 4.1 There is a universsal constant $c>1$ such that the following holds. Suppose that $G$ is primitive but not highly set-transitive. Then

- $f_{n}(G) \geq c^{n} / p(n)$, for some polynomial $p$;

- $F_{n}(G) \geq n ! c^{n} / p(n)$, for some polynomial $p$.

In other words, for $\left(f_{n}\right)$, there is a gap between constant and exponential growth! Merola's proof gives the result with $c=1.324 \ldots$; the best-known examples have $c=2$ (these are circular versions of the rationals partitioned into two dense subsets, that we met earlier).

The main problems for exponential growth are: 
- prove that the "exponential constant" $\lim _{n \rightarrow \infty}\left(f_{n}\right)^{1 / n}$ always exists;

- find the possible values it can take;

- find a structural description of the examples where the growth is no faster than exponential.

What happens just above exponential growth? Here are some examples.

- Let $\mathcal{C}$ be the Fraïssé class each of whose members is a set with a pair of total orders, and $G$ the automorphism group of its Fraïssé limit. Then $f_{n}(G)=n$ ! and $F_{n}(G)=(n !)^{2}$.

- The group $S \mathrm{Wr} S_{2}$ with the power action is primitive. I do not know what the asymptotic behaviour of $\left(f_{n}(G)\right)$ is. As explained earlier, we find that

$$
F_{n}(G) \sim \frac{1}{2} B(n)(B(n)+1),
$$

where $B(n)$ is the Bell number.

- For the permutation group induced by $S$ on the set of 2-element subsets of the domain, we have

$$
F_{n}(G) \sim B(2 n) 2^{-n} n^{-1 / 2} \exp \left(-\left[\frac{1}{2} \log (2 n / \log n)\right]^{2}\right) .
$$

No clear evidence of a gap emerges from this limited data. Macpherson [26] has proved some theorems connecting growth just faster than exponential with model-theoretic properties such as stability and the strict order property.

For the automorphism group of the random graph, the growth rate is about $\exp \left(c n^{2}\right)$. For such growth, it doesn't make a lot of difference whether we consider $F_{n}$ or $f_{n}$. We make one brief observation about this case.

Proposition 4.2 Let $G$ be the automorphism group of a hoomogeneous structure over a finite relational language. Then $F_{n}(G)$ is bounded above by the exponential of a polynomial.

The converse of this is not true. For example, the general linear group on a vector space of countable dimension over the field of two elements has $F_{n}(G)$ roughly $2^{n^{2} / 16}$, but is not the automorphism group of a homogeneous structure over a finite relational language. (Take two $n$-tuples of vectors, 
the first linearly independent and the second satisfying the single linear relation that the sum of the vectors is zero. These two $n$-tuples cannot be distinguished by relations of arity less than $n$.)

An open problem here is to understand what the implications for $G$ are of being the automorphism group of a homogeneous structure over a finite relational language.

\section{Graded algebras}

Another part of mathematics where sequences of positive integers occur is the theory of graded algebras. Such an algebra is a direct sum

$$
A=\bigoplus_{n \in \mathbb{N}} A_{n}
$$

where the $A_{n}$ are vector spaces over a field $F$, and, if $v \in A_{m}$ and $w \in A_{m}$, then the algebra product $v w$ belongs to $A_{m+n}$. The subspaces $A_{n}$ are the homogeneous components. If they are all finite-dimensional, then the sequence of their dimensions (or its ordinary generating function) is the Hilbert series of the algebra. If a graded algebra is finitely generated, then the dimensions grow no faster than a polynomial in $n$, and so the Hilbert series converges inside the unit circle.

We construct graded algebras as follows. First, let $\Omega$ be an infinite set, and $F$ a field (for our purposes, always of characteristic zero). Let $A_{n}$ be the vector space of functions from $\left(\begin{array}{l}\Omega \\ n\end{array}\right)$ (the set of $n$-element subsets of $\Omega$ ) to $F$, and define a multiplication on the homogeneous components by the rule

$$
f g(X)=\sum_{Y \in\left(\begin{array}{l}
X \\
n
\end{array}\right)} f(Y) g(X \backslash Y)
$$

for $f \in A_{n}, g \in A_{m}$, and $X \in\left(\begin{array}{c}\Omega \\ n+m\end{array}\right)$. Extended linearly to $A=\bigoplus A_{n}$, this multiplication is commutative and associative, annd makes $A$ a graded algebra. The constant function 1 on $\left(\begin{array}{c}\Omega \\ 0\end{array}\right)=\{\emptyset\}$ is the identity. (In fact $A_{0}$ is 1-dimensional, spanned by the identity.) This algebra is the reduced incidence algebra of the poset of finite subsets of $\Omega$, but we make no use of this fact.

The algebra $A$ is much too large: its homogeneous components have infinite dimension (except for $A_{0}$ which has dimension 1), and there are many nilpotent elements. 
But it has one important feature: if $e \in A_{1}$ is the constant function on $\Omega$ with value 1 , then $e$ is not a zero-divisor; that is, multiplication by $e$ is a monomorphism from $A_{n}$ to $A_{n+1}$. (This algebraic statement is really a fact about finite combinatorics: the incidence matrix of $n$-sets and $(n+1)$-sets of a set $X$ of cardinality at least $2 n+1$ has full rank.)

Now let $G$ be a permutation group on $\Omega$. There is a natural action of $G$ on $A_{n}$, for all $n$; we let $A_{n}^{G}$ be the set of functions invariant under $G$ (that is, constant on the $G$-orbits), and $A^{G}=\bigoplus A_{n}^{G}$. Then $A^{G}$ is a graded sunalgebra of $A$. Moreover, if $G$ is oligomorphic, then $\operatorname{dim}\left(A_{n}^{G}\right)=f_{n}(G)$, so that the Hilbert series of $A^{G}$ is $f_{G}(x)$.

There are varions interesting examples of groups for which $A^{G}$ is finitely generated. For example, if $G=G_{1} \times G_{2}$ in its intransitive action on $\Omega_{1} \cup \Omega_{2}$, where $G_{i}$ acts on $\Omega_{i}$ for $i=1,2$, we have

$$
A^{G} \cong A^{G_{1}} \otimes_{F} A^{G_{2}}
$$

for any field of characteristic zero. In particular, $A^{S \times S}$ is freely generated by (that is, a polynomial ring in) two generators of degree 1, and more generally, $A^{S^{r}}$ freely generated by $r$ generators of degree 1 .

If $H$ is a finite permutation group of degree $n$, and $G=S \mathrm{Wr} H$ (in its imprimitive action), then $A^{G}$ is isomorphic to the ring of invariants of $H$ (acting as a linear group via permutation matrices). In particular, if $H=S_{n}$, then $A^{G}$ is the ring of symmetric polynomials in $n$ variables, and is freely generated by the elementary symmetric polynomials $e_{1}, \ldots, e_{n}$ of degrees $1,2, \ldots, n$, by Newton's Theorem.

However, it follows from Macpherson's Theorem that, if $G$ is primitive but not highly homogeneous, then $A^{G}$ cannot be finitely generated.

In the light of this, we are forced to look for other kinds of algebraic properties of $A^{G}$. An example is a remarkable recent theorem of Pouzet [30], confirming a 30-year-old conjecture:

Theorem 5.1 Let $G$ be a group with no finite orbits. Then $A^{G}$ is an integral domain if and only if $G$ has no finite orbits.

One direction is trivial: if $f \in A_{n}$ is the characteristic function of a $G$ orbit, then $f \in A_{n}^{G}$ and $f^{2}=0$. The converse requires a new type of Ramseytype theorem which is likely to have further applications. The result itself is applicable to growth rates, in view of the following result [6], whose proof uses some easy dimension theory from algebraic geometry: 
Theorem 5.2 If $G$ is oligomorphic and $A^{G}$ is an integral domain then $f_{m+n} \geq$ $f_{m}+f_{n}-1$.

Another situation in which we can describe the structure of $A^{G}$ is when $G$ is associated with a Fraïssé class $\mathcal{C}$ (that ism $G$ is the automorphism group of the Fraïssé limit of $\mathcal{C}$ ). In [5] it is shown that, if the class $\mathcal{C}$ has notions of connected components, disjoint union, and spanning substructures satisfying a few simple axioms, then $A^{G}$ is a polynomial algebra whose generators are the characteristic functions of the connected structures in $\mathcal{C}$. Here are a few, reasonably typical, examples.

- Let $\mathcal{C}$ be the class of finite graphs, so that $G$ is the automorphism group of the random graph $R$. Then a basis for the $n$th homogeneous component of $A^{G}$ consists the characteristic functions of all $n$-vertex graphs (so that $\operatorname{dim}\left(A_{n}^{G}\right)=f_{n}(G)$ is the number of unlabelled graphs on $n$ vertices). Now the characteristic functions of connected graphs on $n$ vertices are algebraically independent, and the set of all such elements forms a free generating set for $A^{G}$, which is a polynomial algebra (infinitely generated).

- We saw earlier that, if $G$ is associated with a Fraïssé class $\mathcal{C}$, then $G \mathrm{Wr} S$ is associated with the class of disjoint unions of $\mathcal{C}$-structures. There is an obvious notion of connectedness: structures with a single part are connected! Thus, $A^{G \mathrm{Wr} S}$ is a polynomial algebra, with $f_{n}(G)$ generators of degree $n$ for all $n$. (Thus, the structure of $A^{G \mathrm{Wr} S}$ is independendent of the structure of $A^{G}$ except for numerical information about dimensions of components.)

- Consider the set $\mathbb{Q}$ with two kinds of structure: the order, and $m$ subsets forming a partition of $\mathbb{Q}$, each one dense in $\mathbb{Q}$. (Think of a colouring of $\mathbb{Q}$ with $m$ colours $c_{1}, c_{2}, \ldots, c_{m}$, so that each colour class is dense.) Let $G$ be the group of permutations preserving the order and the colours. An orbit of $G$ on $n$-sets is described uniquely by a word of length $n$ in the alphabet $\left\{c_{1}, c_{2}, \ldots, c_{m}\right\}$, recording the colours of the elements of the set in increasing order. Now the algebra $A^{G}$ is the shuffle algebra over an alphabet of size $m$, which occurs in the theory of free Lie algebras [32]. One can develop an appropriate notion of connectedness, so that the connected words are the so-called Lyndon words. The fact that the shuffle algebra is a polynomial algebra 
generated by the Lyndon words was first proved by Radford [31], but emerges as a special case of the theory presented here.

\section{Group structure}

Relatively little is known about the structure of closed oligomorphic permutation groups, but the picture is rapidly changing. We look first at the normal structure in a couple of classical examples.

The countable symmetric group $S=\operatorname{Sym}(\Omega)$ has a normal subgroup $\operatorname{FSym}(\Omega)$, the finitary symmetric group, consisting of all permutations moving only finitely many points; this has a normal subgroup of index 2 , the alternating group $\operatorname{Alt}(\Omega)$, consisting of finitary permutations which are even permutations of their supports. These are the only non-trivial proper normal subgroups; in particular, the quotients are simple.

The group $A$ of order-preserving permutations of $\mathbb{Q}$ has two normal subgroups $L$ and $R$; $L$ consists of all permutations fixing all sufficiently large positive rationals, and $R$ consists of permutations fixing all sufficiently large negative rationals. Their intersection consists of the order-preserving permutations of bounded support. These are the only non-trivial normal subgroups. In particular, $A /(L \cap R) \cong L /(L \cap R) \times R /(L \cap R)$, and the two factors are isomorphic.

The automorphism group of the countable random graph is simple [35]. Indeed, given any two elements $g, h$ of this group with $g \neq 1$, it is possible to write $h$ as the product of three copies of $g$ or $g^{-1}$.

An important property which has had a lot of attention is the small index property. Let $G$ be a permutation group of countable degree. A subgroup $H$ has small index if $|G: H|<2^{\aleph_{0}}$. (If the Continuum Hypothesis holds, this just says that $H$ has finite or countable index.) The stabiliser of any finite set has small index. We say that $G$ has the small index property if any subgroup of $G$ of small index contains the pointwise stabiliser of a finite set; it has the strong small index property if every subgroup of small index lies between the pointwise and setwise stabilisers of a finite set. If $G$ is a closed oligomorphic group with the small index property, then the topology of $G$ is determined by the group structure: a subgroup is open if and only if it has small index, so the subgroups of small index form a neighbourhood basis of the identity.

The symmetric group $S$, the group $A$ of order-preserving permutations 
of $\mathbb{Q}$, and the automorphism group of the random graph all have the strong small index property $[15,19,8]$. A typical example of a permutation group which does not have the strong small index property is $S \mathrm{Wr} S$, in its imprimitive action; the stabiliser of a block of imprimitivity is not contained in the setwise stabiliser of any finite set. Examples which do not have the small index property can be constructed by producing automorphism groups of Fraïssé limits which have infinite elementary abelian 2-groups as quotients; they have "too many" subgroups of small index!

I mentioned earlier that the notion of Baire category is important for closed oligomorphic groups. Such a group $G$ is said to have generics if there is a conjugacy class which is residual in $G$. In each of the three groups mentioned above, generics exist. Indeed, the $n$th direct power of the group has generics (in other words, the original group has generic $n$-tuples) for all $n$. This property is closely related to the small index property (see [19]).

We can also ask what group is generated by a "tylical" $n$-tuple of elements. John Dixon proved that almost all pairs of elements of the finite symmetric group $S_{n}$ generate $S_{n}$ or the alternating group $A_{n}$. Later [13], he proved an analogue for the symmetric group of countable degree: almost all pairs of elements (in the sense of Baire category, that is, a residual set) generate a highly transitive free subgroup of $S$. (The existence of highly transitive free groups was first shown by McDonough [28].)

As noted, very recently these results have been extended to wider classes of groups.

Macpherson and Tent define the free amalgamation property, which is a strengthening of the strong amalgamation property, as follows. A Fraïssé class $\mathcal{C}$ has the free amalgamation property if, whenever $B_{1}$ and $B_{2}$ are structures in $\mathcal{C}$ with a common substructure $A$, there is an amalgam $C$ of $B_{1}$ and $B_{2}$ such that

- the intersection of $B_{1}$ and $B_{2}$ in $C$ is precisely $A$ (this is the content of strong amalgamation, see Section 2;

- Every instance of a relation in $C$ is contained in either $B_{1}$ or $B_{2}$.

This holds for graphs: we can choose to make the amalgam so that there are no edges between $B_{1} \backslash A$ and $B_{2} \backslash A$.

They prove the following theorem:

Theorem 6.1 Let $\mathcal{C}$ be a nontrivial Fraïssé class (that is, there are some 
non-trivial relations) with the free amalgamation property, and $G$ the automorphism group of its Fraïsé limit. Then $G$ is simple.

The trivial case must be excluded, since then $G$ is the symmetric group $S$, and as we have seen this group is not simple.

Gartside and Knight [17] say that a Polish group (a topological group whose topology comes from a complete metric) is almost free if a residual subset of the $n$-tuples of elements of $G$ freely generate a free group. They give a number of equivalent characterisations of such groups, and show:

Theorem 6.2 Closed oligomorphic groups are almost free.

Kechris and Rosendal [23] give conditions for the existence of generic conjugacy classes in a closed oligomorphic group.

In closing I mention a couple of important papers linking oligomorphic permutation groups, dynamical systems, and Ramsey theory: [20, 22].

\section{References}

[1] R. Applegate and P. J. Cameron, Orbits on $n$-tuples, Communications in Algebra, to appear.

[2] R. A. Bailey, Association Schemes: Designed Experiments, Algebra and Combinatorics, Cambridge University Press, Cambridge, 2004.

[3] P. J. Cameron, Transitivity of permutation groups on unordered sets, Math. Z. 48 (1976), 127-139.

[4] P. J. Cameron, The random graph, pp. 331-351 in The Mathematics of Paul Erdős (ed. R. L. Graham and J. Nesetril), Springer, Berlin, 1997.

[5] P. J. Cameron, The algebra of an age, in Model Theory of Groups and Automorphism Groups (ed. David M. Evans), London Mathematical Society Lecture Notes 244, Cambridge University Press, Cambridge, 1997, pp. 126-133.

[6] P. J. Cameron, On an algebra related to orbit-counting, J. Group Theory 1 (1998), 173-179. 
[7] P. J. Cameron, Permutation Groups, London Math. Soc. Student Texts 45, Cambridge University Press, Cambridge, 1999

[8] P. J. Cameron, The random graph has the strong small index property, Discrete Math. 291 (2005), 41-43.

[9] P. J. Cameron, D. Gewurz and F. Merola, Product action, Discrete Math. 308 (2008), 386-394.

[10] P. J. Cameron, T. Prellberg and D. Stark, Asymptotic enumeration of incidence matrices, Journal of Physics: Conference Series 42 (2006), $59-70$.

[11] P. J. Cameron, T. Prellberg and D. Stark, Asymptotic enumeration of 2-covers and line graphs, preprint.

[12] P. J. Cameron and D. E. Taylor, Stirling numbers and affine equivalence, Ars Combinatoria 20B (1985), 3-14.

[13] J. D. Dixon, Most finitely generated permutation groups are free. textitBull. London Math. Soc. 22 (1990), 222-226.

[14] J. D. Dixon and B. Mortimer, Permutation Groups, Springer, New York, 1996

[15] J. D. Dixon, P. M. Neumann, and S. Thomas, Subgroups of small index in infinite symmetric groups, Bull. London Math. Soc. 18 (1986), 580586.

[16] A. Dress and T. W. Müller, Decomposable functors and the exponential principle, Advances in Math. 129 (1997), 188-221.

[17] P. M. Gartside and R. W. Knight, Ubiquity of free subgroups, Bull. London Math. Soc. 35 (2003), 624-634.

[18] I. P. Goulden and D. M. Jackson, Combinatorial Enumeration, Dover Publ. (reprint), new York, 2004.

[19] W. A. Hodges, I. Hodkinson, D. Lascar and S. Shelah, The small index property for $\omega$-stable $\omega$-categorical structures and for the random graph. J. London Math. Soc. (2) 48 (1993), 204-218. 
[20] J. Hubička and J. Nešetřil, Finite presentation of homogeneous graphs, posets and Ramsey classes, Israel J. Math. 149 (2005), 21-44.

[21] A. Joyal, Une theorie combinatoire des séries formelles, Adv. Math. 42 (1981), 1-82.

[22] A. S. Kechris, V. G. Pestov and S. B. Todorčević, Fraïssé limits, Ramsey theory, and topological dynamics of automorphism groups, Geometric and Functional Analysis 15 (2005), 106-189.

[23] A. S. Kechris and C. Rosendal, Turbulence, amalgamation, and generic automorphisms of homogeneous structures, Proc. Lond. Math. Soc. (3) 94 (2007), 302-350.

[24] A. H. Lachlan and R. E. Woodrow, Countable ultrahomogeneous undirected graphs, Trans. Amer. Math. Soc. 262 (1980), 51-94.

[25] H. D. Macpherson, The action of an infinite permutation group on the unordered subsets of a set, Proc. London Math. Soc. (3) 46 (1983), 471486.

[26] H. D. Macpherson, Permutation groups of rapid growth, J. London Math. Soc. (2) 35 (1987), 276-286.

[27] H. D. Macpherson and K. Tent, Simplicity of some automorphism groups, preprint.

[28] T. P. McDonough, A permutation representation of a free group, Quart. J. Math. Oxford (2) 28 (1977), 353-356.

[29] F. Merola, Orbits on $n$-tuples for infinite permutation groups, Europ. J. Combinatorics 22 (2001), 225-241.

[30] M. Pouzet, When the orbit algebra of a group is an integral domain? Proof of a conjecture of P. J. Cameron, to appear.

[31] D. E. Radford, A natural ring basis for the shuffle algebra and an application to group schemes, J. Algebra 58 (1979), 432-454.

[32] C. Reutenauer, Free Lie Algebras, London Math. Soc. Monographs (New Series) 7, Oxford University Press, Oxford, 1993. 
[33] R. P. Stanley, Generating functions, in: MAA Studies in Mathematics, vol. 17 (G.-C. Rota ed.), Mathematical Association of America, Washington, 1978, pp. 100-141.

[34] S. R. Thomas, Reducts of the random graph, J. Symbolic Logic 56 (1991), 176-181.

[35] J. K. Truss, The group of the countable universal graph, Math. Proc. Cambridge Philos. Soc. 98 (1985), 213-245. 